\title{
Lidil
}

Revue de linguistique et de didactique des langues

\section{L'oral dans la classe. Compétences, enseignement, activités}

HALTÉ J.-F., RISPAIL M. (éd.) (2005), L'Harmattan, 297 p.

\section{Christian Degache}

\section{(2) OpenEdition}

Journals

Édition électronique

URL : http://journals.openedition.org/lidil/122

DOI : $10.4000 /$ lidil. 122

ISSN : 1960-6052

Éditeur

UGA Éditions/Université Grenoble Alpes

Édition imprimée

Date de publication : 1 décembre 2005

ISBN : 2-914176-13-9

ISSN : $1146-6480$

Référence électronique

Christian Degache, «L'oral dans la classe. Compétences, enseignement, activités », Lidil [En ligne], 32 | 2005, mis en ligne le 18 juillet 2007, consulté le 10 décembre 2020. URL : http:// journals.openedition.org/lidil/122 ; DOI : https://doi.org/10.4000/lidil.122

Ce document a été généré automatiquement le 10 décembre 2020.

(C) Lidil 


\title{
L'oral dans la classe. Compétences, enseignement, activités
}

\author{
HALTÉ J.-F., RISPAIL M. (éd.) (2005), L'Harmattan, 297 p.
}

\section{Christian Degache}

1 Dans cet ouvrage collectif, Jean-François Halté et Marielle Rispail rassemblent et présentent une partie des communications à orientation didactique du colloque «Le langage oral de l'enfant scolarisé : acquisition, enseignement, remédiation ", qui s'est tenu à l'IUFM de Grenoble du 23 au 25 octobre 2003. L'objectif général de ce colloque était de favoriser la réflexion et les échanges autour des domaines de l'acquisition et de la didactique de l'oral, entre diverses communautés de chercheurs et de praticiens.

Dans quel but, comment, avec qui, de quoi, quand parle-t-on en classe? Qu'est-ce qui est en jeu dans l'échange entre enseignants et élèves? Qu'est-ce qui change d'un objet de dialogue à un autre ? Par ailleurs, l'oral doit-il être enseigné ? Et si oui, quel oral ? Sous quelle forme? Avec quel rapport à l'écrit? Telles sont quelques-unes des questions abordées ici.

3 Les coordinateurs ont procédé à des choix judicieux quant à l'organisation de l'ouvrage. Le texte de Jean-François Halté, placé en ouverture, donne la tonalité en traçant et justifiant les grandes lignes de ce que devrait être une véritable didactique des activités langagières, tant sur le plan de la recherche que des pratiques d'enseignement, intégrant oral et écrit dans un ensemble cohérent. Les 18 contributions qui suivent, organisées en "chapitres", sont ensuite regroupées en quatre parties. La $1^{\text {re }}$, intitulée "Quel oral?", réunit les contributions qui, à travers des approches théoriques et pratiques diverses, posent la question de la définition de l'oral en classe. La $2^{\mathrm{e}}$ partie, «Étudier l'oral » donne l'occasion de présenter quelques questions essentielles dans la recherche sur l'oral. Enfin, les deux dernières parties, «La communication orale » et "Oral et apprentissages", rassemblent les textes qui traitent des fonctions de l'oral dans la classe. Ainsi, ce recueil d'articles dessine un itinéraire allant des aspects conceptuels et définitoires de l'oral à ses dimensions communicatives et cognitives en abordant au passage la question des outils nécessaires pour la recherche. 
Rassemblés autour de questions transversales, les 18 chapitres abordent différentes situations d'apprentissage. Les dénominateurs communs de l'ouvrage sont l'oral et la classe certes, mais aussi l'enfant, conformément au titre du colloque à l'origine de ce recueil d'articles. En effet, même si le mot n'apparaît plus dans le titre, la grande majorité des contributions concernent l'enfant scolarisé en primaire (écoles maternelle et élémentaire en France, jusqu'à l'âge de 11 ans, y compris une classe d'enfants sourds de $3^{\text {e }}$ année d'école élémentaire) ou ses enseignants (chap. 8 et 2). Seul le travail de Pambou (chap.17) porte sur des enfants du collège, alors que celui d'Idiazabal et Larringan (chap. 5) concerne, dans une perspective comparative, les adultes, enfants et adolescents. La diversité des activités et des disciplines scolaires concernées, faisant l'objet d'interactions verbales, est importante: l'exposé, la production d'une règle mathématique, la compréhension d'un texte, le débat, les entretiens du matin en maternelle, l'explication de petits travaux d'expérimentation scientifique, la découverte de l'anglais langue étrangère, le dialogue pour comprendre des énoncés écrits et des textes informatifs en histoire, les ateliers de philosophie avec des enfants de 6-7 ans, des moments de classe « ordinaires " (l'affichage de la date en maternelle), les ateliers de verbalisation (entretien professeur-élève), la dictée à l'adulte, la réflexion grammaticale, les activités de découverte du monde (la châtaigne en botanique), la narration d'un événement vécu, diverses activités centrées sur la langue ou non en CLIN (Classe d'initiation au français)... Le fil conducteur est celui des interactions verbales de la classe, des interactions entre professeurs et élèves essentiellement. Mais toutes les contributions ne se proposent pas de les analyser. Cortier (chap. 2), par exemple, travaille sur les représentations des enseignants sur l'oral, Le Manchec et Totereau (chap. 6) sur une étude préliminaire à l'élaboration de grilles d'observation pour permettre aux enseignants de pratiquer l'évaluation diagnostique du langage oral à l'école maternelle.

5 Suivant ses intérêts, le lecteur pourra donc s'orienter vers l'un ou l'autre chapitre après avoir identifié activité et contexte, ce qui suppose toutefois, en l'absence de résumé introductif des chapitres, d'en faire une lecture «diagonale ». Ce faisant, le lecteur pourra aussi se laisser séduire par un chapitre voisin, portant sur une autre activité, un autre contexte, puisque l'ouvrage ne cloisonne pas les contributions selon ces catégories. C'est là toute la force de l'ouvrage. Les coordinateurs ont en effet su intégrer le tout dans un ensemble cohérent, et ce, grâce à divers procédés : par des choix heureux en termes de structuration de l'ouvrage comme nous l'avons signalé; mais aussi par la clarté théorique de l'article introductif de J.-F. Halté et la justesse de son état des lieux sans concessions ; enfin, grâce à une justification pertinente des choix des deux coordinateurs dans un petit article d'introduction qui sait susciter l'envie de lire l'ensemble des contributions de l'ouvrage. On y trouve notamment la justification d'un autre aspect saillant de l'ouvrage, qui contribue à établir une forte cohérence entre le fond et la forme, à savoir le choix de faire figurer en introduction d'un nombre important de chapitres de larges extraits des corpus analysés « pour mettre en valeur la parole des élèves et en montrer l'aspect premier et irréductible» (p. 10): 5 chapitres (sur 18) sont concernés, ce qui ne constitue pas la majorité mais est suffisamment rare pour être souligné, d'autant que 5 autres chapitres s'appuient sur de larges extraits de corpus à l'intérieur des textes. On peut donc dire que l'intention d'ancrer l'ouvrage dans la matérialité verbale des interactions se concrétise au fil des chapitres. Il reste que les coordinateurs sont bien conscients des limites de la méthodologie empirique de recherche en "situation dite "écologique" " qui caractérise l'ensemble des 
contributions du présent ouvrage (p.10). Mais comme ils le précisent, il s'agissait d'éviter les « "discours sur" qui font peut-être obstacle à la rencontre entre enseignants et chercheurs ", et en cela ils ont parfaitement réussi. C'est en effet avec un plaisir sans cesse renouvelé que l'on passe d'une situation d'apprentissage à une autre et que l'on découvre, à travers les extraits de corpus et les descriptions que font les auteurs, toute la diversité et l'importance des productions langagières orales dans la classe.

Une autre originalité de l'ouvrage est de faire figurer la définition la plus convaincante de l'oral dans le chapitre sur les enfants sourds de Mugnier et Millet (chap. 4). Situé à la fin de la 1re partie "quel oral?", les auteures y prennent appui sur une définition anthropologique pour clarifier la polysémie de la notion d'oral à travers la prise en compte de trois critères fondamentaux de l'oralité : le fait que l'oral suppose une acquisition naturelle; le rôle déterminant du contexte dans la construction du sens en situation de communication tout au long de l'acquisition ; la primauté de la dimension interactive. Si l'on accepte de ne plus confondre langue orale et langue vocale, parce que l'oralité est attestée aussi bien dans la langue vocale que dans la langue gestuelle, alors il devient possible d'appréhender le caractère essentiel de l'oral. Comme souvent, c'est par contraste et grâce à l'interdisciplinarité, que l'essence même de l'objet observé et analysé nous apparait.

7 Les notions d'oralité et de scripturalité, en tant qu'ordres discursifs, apparaissent comme récurrentes au fil des chapitres. Dès son article de cadrage, Halté propose (p. 26 et $s q$.$) de s'orienter vers une didactique des activités langagières susceptible de les$ intégrer. Cette proposition, dont il fait le titre de sa contribution, est reprise par plusieurs auteurs dans l'ouvrage. Elle étonnera en revanche le lecteur familiarisé avec le Cadre européen commun de référence pour les langues². Cet ouvrage, conçu pour l'enseignement, l'apprentissage et l'évaluation des langues, qu'elles soient étrangères, secondes ou maternelles, constitue déjà un traité de didactique des activités langagières puisqu'il s'emploie à les définir dans le détail, en relation aux habiletés, tâches et niveaux de compétence. L'oral, que ce soit en production, compréhension, interaction ou médiation, y tient d'ailleurs une place de choix. Pourquoi dès lors ne pas s'en inspirer pour la didactique des langues dites maternelles ${ }^{3}$ ?

8 On ne pourra pas cependant regretter que les langues étrangères soient absentes des problématiques abordées dans les différents chapitres de «L'oral dans la classe », bien au contraire. Chanfrault-Duchet (chap. 1) s'inspire d'une démarche intégrant l'oralité en didactique de l'anglais langue étrangère. Cortier (chap. 2, p. 55) remarque que le travail de l'oral pour l'oral correspond à « des activités jusqu'ici davantage développées dans le cadre de l'enseignement des langues étrangères ». Et surtout, les chapitres 9, 17 et 18 portent sur des problématiques de l'apprentissage de l'anglais langue étrangère (en grande section de maternelle) ou du français langue seconde (au Gabon et en classe d'intégration en France). Mais en dépit de ces mentions, les apports de la didactique des langues ne semblent pas avoir été pris en compte dans la problématique globale de l'ouvrage et il est permis de le regretter. Sans doute peut-on voir là une perspective de prolongement de ces travaux. On prendra à ce sujet les derniers mots du livre comme un signe de ce nécessaire rapprochement des didactiques des langues étrangères et maternelles autour de l'oral en classe, lorsque Vasseur présente l'apprentissage sociodiscursif comme une démarche à «favoriser et encourager dans les CLIN comme dans les classes de langue étrangère en général ». 
Une autre perspective de prolongement peut se situer au niveau de la notion de classe. Dans cet ouvrage, seule la configuration présentielle classique de l'enseignant devant ses élèves, est prise en compte. Certes la diversité des classes considérées donne à l'ouvrage une richesse importante. Mais il est permis de penser que l'étude de la notion même d'oralité pourrait être mieux circonscrite si l'on prenait en compte des situations de classes non-présentielles, par exemple pour dégager l'oralité présente dans les interactions synchrones et asynchrones écrites en ligne (clavardage, forum, courriels...).

Au total, J.-F. Halté et M. Rispail nous offrent là un utile tour d'horizon, un ouvrage de référence à partir duquel il sera possible de lancer d'autres recherches. Chercheurs et praticiens trouveront intérêt à consulter cet ouvrage, beau témoignage d'interdisciplinarité et du rapprochement nécessaire entre didacticiens de tous bords autour de questions langagières transversales.

\section{NOTES}

1. <http://www.grenoble.iufm.fr/kiosque/colloqueLang.htm>

2. Collectif (2001), Cadre européen commun de référence pour les langues. Apprendre, enseigner, évaluer, Conseil de la Coopération culturelle, Comité de l'éducation «Apprentissage des langues et citoyenneté européenne », Division des langues vivantes, Conseil de l'Europe, Strasbourg, Didier.

3. On considérera à ce titre comme symptomatique le fait que le «Portfolio européen des langues, version collège ", dans une version destinée aux jeunes francophones, intègre dans la partie « Ma biographie langagière » dédiée à l'auto-évaluation de leurs savoir-faire en langues étrangères, une colonne - la dernière - intitulée « et en français » (Castellotti, Coste, Moore, Tagliante, 2003, Didier).

\section{AUTEUR}

\section{CHRISTIAN DEGACHE}

Université Stendhal-Grenoble 3, Laboratoire Lidilem. 